\title{
Double-Time-Scale Coordinated Voltage Control in Active Distribution Networks Based
} on MPC

\author{
Guo, Yifei; Wu, Qiuwei; Gao, Houlei; Huang, Sheng; Zhou, Bin; Li, Canbing
}

Published in:

IEEE Transactions on Sustainable Energy

Link to article, DOI:

10.1109/TSTE.2018.2890621

Publication date:

2020

Document Version

Peer reviewed version

Link back to DTU Orbit

Citation (APA):

Guo, Y., Wu, Q., Gao, H., Huang, S., Zhou, B., \& Li, C. (2020). Double-Time-Scale Coordinated Voltage Control in Active Distribution Networks Based on MPC. IEEE Transactions on Sustainable Energy, 11(1), 294 - 303. https://doi.org/10.1109/TSTE.2018.2890621

\section{General rights}

Copyright and moral rights for the publications made accessible in the public portal are retained by the authors and/or other copyright owners and it is a condition of accessing publications that users recognise and abide by the legal requirements associated with these rights.

- Users may download and print one copy of any publication from the public portal for the purpose of private study or research.

- You may not further distribute the material or use it for any profit-making activity or commercial gain

- You may freely distribute the URL identifying the publication in the public portal 


\title{
Double-Time-Scale Coordinated Voltage Control in Active Distribution Networks Based on MPC
}

\author{
Yifei Guo, Qiuwei Wu, Senior Member, IEEE, Houlei Gao, Member, IEEE, Sheng Huang, \\ Bin Zhou, Senior Member, IEEE, and Canbing Li, Senior Member, IEEE
}

\begin{abstract}
This paper proposes a double-time-scale coordinated voltage control scheme for distribution networks with distributed generators (DGs) based on Model Predictive Control (MPC) to regulate the voltage profile across a network. The slow-timescale control (STC) scheme is designed to correct the long-term voltage deviations while reducing the number of actions of the on-load tap changer (OLTC), step voltage regulators (SVRs) and capacitor banks (CBs). The MPC problem is formulated as a mixed-integer quadratic programming (MIQP). A tailored exaction solution method based on the Branch-and-Bound (B\&B) algorithm embedded with an Alternating Direction Method of Multipliers (ADMM)-based QP solver is developed to efficiently solve the MIQP problem. In the fast-time-scale control (FTC), the active and reactive power outputs of DGs are optimally coordinated to handle the fast voltage fluctuations as well as capture more renewable energy. An efficient analytical sensitivity calculation method is used to update the voltage sensitivities online. The effectiveness of the proposed control scheme along with the exact solution method is verified on a modified real 20 $\mathrm{kV}$ distribution system.
\end{abstract}

Index Terms-Active distribution network, alternating direction method of multipliers (ADMM), distributed generator (DG), mixed-integer quadratic programming (MIQP), model predictive control (MPC), voltage control.

\section{NOMENCLATURE}

\section{A. Abbreviations}

$\begin{array}{ll}\text { ADMM } & \text { Alternating direction method of multipliers } \\ \text { B\&B } & \text { Branch and bound } \\ \text { CB } & \text { Capacitor bank } \\ \text { DG } & \text { Distributed generator }\end{array}$

This work was supported in part by the ForskEL program through the 'IDE4L-DK Top-Up' project, in part by National Natural Science Foundation of China under grant 51877127 and in part by the China Scholarship Council (CSC). (Corresponding author: Qiuwei Wu.)

Y. Guo is with Key Laboratory of Power System Intelligent Dispatch and Control of Ministry of Education, Shandong University, Jinan 250061, China and Center for Electric and Energy (CEE), Department of Electrical Engineering, Technical University of Denmark (DTU), Kgs. Lyngby 2800, Denmark (e-mail:yfguo.sdu@gmail.com).

Q. Wu is with the Centre for Electric Power and Energy, Department of Electrical Engineering, Technical University of Denmark, Lyngby, 2800, Denmark and the Harvard China Project, School of Engineering and Applied Sciences, Harvard University, 29 Oxford Street, Cambridge, MA 02138, USA (e-mail: qw@elektro.dtu.dk,qiuwu@seas.harvard.edu).

H. Gao is with Key Laboratory of Power System Intelligent Dispatch and Control of Ministry of Education, Shandong University, Jinan 250061, China (e-mail:houleig@sdu.edu.cn).

S. Huang is with the Center for Electric and Energy (CEE), Department of Electrical Engineering, Technical University of Denmark (DTU), Kgs. Lyngby 2800, Denmark (e-mail:huang98123@163.com).

B. Zhou and C. Li are with the College of Electrical and Information Engineering, Hunan University, Changsha 410082, China (email:binzhou@hnu.edu.cn;lcb@hnu.edu.cn).

$\begin{array}{ll}\text { FTC } & \text { Fast-time-scale control } \\ \text { MPC } & \text { Model predictive control } \\ \text { MIQP } & \text { Mixed-integer quadratic programming } \\ \text { OLTC } & \text { On-load tap changer } \\ \text { OPF } & \text { Optimal power flow } \\ \text { PV } & \text { Photovoltaic } \\ \text { STC } & \text { Slow-time-scale control } \\ \text { SVR } & \text { Step voltage regulator }\end{array}$

\section{INTRODUCTION}

T NTEGRATION of renewables-based distributed generators (DGs), such as photovoltaic (PV) and wind power, is seen as a promising solution to alleviate reliance on depleting fossil fuel reserves, increase energy security and provide an environment-friendly solution to growing power demand [1]. The increasing penetration of DGs brings a number of technical and economic challenges to distribution network operators. Voltage profile is one of the most notable issues, since the reverse power flow caused by the high production of DGs could result in severe voltage rise [2]. Furthermore, the variability and uncertainty associated with renewables could lead to voltage fluctuations.

Local autonomous control strategies are cost-saving and easy to implement [3]-[4]. Active and reactive power outputs of DGs are adjusted to mitigate the voltage deviations. However, such non-coordination strategies might lead to competition among DGs and might interface with the conventional voltage regulation devices, posing the possibility of oscillation or even undesired islanding [5].

In comparison, optimization-based coordinated voltage control could avoid the drawbacks of local control and achieve optimal operation of systems, consequently motivating a considerable number of studies. These methods can be roughly classified into two categories: optimal power flow (OPF)-based methods [6]-[13] and sensitivity-based methods [14]-[17]. For the first category, the voltage control problems are formulated as a standard OPF problem to achieve multiple goals such as correcting voltage deviations, reducing the power losses, as well as reducing the number of actions of discrete devices including on-load tap changers (OLTCs) of the main transformer, step voltage regulators (SVRs) and capacitor banks (CBs). For the second category, [14]-[17], the sensitivity-based method is essentially a numerical linearization version around the operating point, and consequently constitutes a convex approximation to the original non-convex AC OPF problem, which is less computationally expensive and therefore more suitable 
for real-time control. The distance between the solution of the sensitivity method and the optimal solution based on the standard OPF model proves to be quite small [16].

Despite many studies on voltage control, the existing schemes are mostly designed under one time scale, which might fail to optimally coordinate different voltage regulation devices with different temporal characteristics. The number of the switching operations of discrete device variables is often optimized in the day-ahead planning based on the assumption that day-ahead load and generation forecasting is accurate enough. However, this fails to tackle the real-time uncertain voltage variations in the fast time scale, and the control performance highly relies on forecasting accuracy.

Furthermore, the discrete control variables complicate the problems, resulting in mixed-integer programming problems. For simplification, the discrete variables are generally considered as continuous variables when the problems are solved, and then are rounded to the closest discrete value [8]. In [6], an improved strategy was proposed which compares the closest two discrete values. Moreover, the heuristic search methods such as the particle-swarm-optimization algorithm [11] and the differential evolution algorithm [12] are often used to solve complex problems. However, these methods suffer from suboptimality.

Therefore, the main contributions of this paper are threefold:

- A double-time-scale voltage control framework based on the MPC is proposed. In the slow-time-scale control (STC), the voltage profile and operation of slow-timescale voltage control devices with discrete nature is optimized. The fast-time-scale control (FTC) is designed to deal with the fast voltage issues by coordinating the active and reactive power injections of DGs.

- An analytical voltage-sensitivity computation method w.r.t the discrete settings of voltage regulation devices is proposed by extending the method in [18].

- A tailored solution method combining the Branch-andBound (B\&B) and Alternating Direction Method of Multipliers (ADMM) algorithms is designed to efficiently solve the MPC problem, especially for mixed-integer quadratic programming (MIQP) problems in the STC, guaranteeing the optimality of the solution.

The rest of this paper is organized as follows. Section II briefly introduces the voltage control scheme. Section III presents the MPC problem formulation. Section IV presents the online sensitivity calculation method. The solution method combining B\&B and ADMM algorithms is presented in Section V. Numerical results are presented in Section VI, followed by conclusions.

\section{BRIEF OVERVIEW}

The voltage control devices in distribution networks have different temporal characteristics, which should be addressed in the voltage control system design. The main OLTC transformer in the substation, SVRs and CBs with slow and discrete nature should correspond to the voltage regulation in a time scale of several tens of minutes to several hours. Moreover, from an economic perspective, these devices should

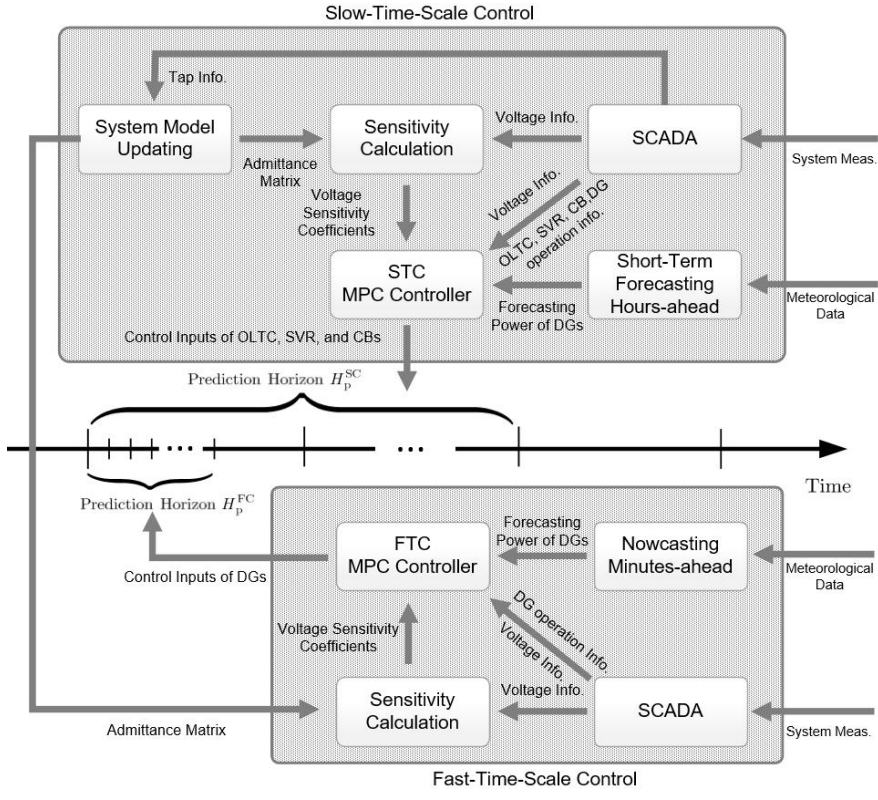

Fig. 1. Architecture of the double-time-scale voltage control scheme.

not have to be frequently adjusted so as to prolong their lifetime. However, power-electronics-interfaced DGs with fast and continuous nature can be used to deal with voltage issues with a time scale of a few seconds to several minutes. Accordingly, these voltage regulation devices should be coordinately operated in different time scales.

In this paper, a centralized double-time-scale voltage control scheme is designed as illustrated in Fig. 1. All the data are gathered by the SCADA system and a central processor makes the control decisions for the whole distribution network [19]. In the STC, the operation of conventional voltage regulation devices including OLTC, SVRs and CBs is optimally coordinated such that the active power of DGs is assumed to track the maximum power point according to the online short-term forecasting. Although the potential reactive power capabilities of DGs are considered, only the conventional devices are scheduled at this level. In the FTC, the active and reactive power outputs of DGs are coordinated to minimize the voltage deviations in the fast time scale. The active power curtailment of DGs is considered as a necessary strategy to correct the severe voltage deviations.

\section{PROBLEM Formulation}

In the MPC, suppose the prediction and control horizon are $H_{p}$ and $H_{c}$, respectively. The prediction and control steps are denoted by $N_{p}=H_{p} / T_{c}$ and $N_{c}=H_{c} / T_{c}$, where $T_{c}$ denotes the control period. Generally, the prediction horizon should be equal to or longer than the control horizon. From a computational viewpoint, we select $N_{p}=N_{c}$, since there are no clear advantages to choose $N_{p}>N_{c}$ [17], [20].

Define the variables in vector form at step $k, \quad \mathbf{n}_{\text {tap }}(k)=\left[n_{\text {tap }, 1}(k), \ldots, n_{\text {tap }, N_{\text {tap }}}(k)\right]^{T}$ for tap position (including the OLTC and SVRs), $\mathbf{n}_{\mathrm{CB}}(k)=\left[n_{\mathrm{CB}, 1}(k), \ldots, n_{\mathrm{CB}, N_{\mathrm{CB}}}(k)\right]^{T}$ for CB settings, $\mathbf{P}_{\mathrm{DG}}(k)=\left[P_{\mathrm{DG}, 1}(k), \ldots, P_{\mathrm{DG}, N_{\mathrm{DG}}}(k)\right]^{T}$ for active power 
injections of DGs, $\mathbf{Q}_{\mathrm{DG}}(k)=\left[Q_{\mathrm{DG}, 1}(k), \ldots, Q_{\mathrm{DG}, N_{\mathrm{DG}}}(k)\right]^{T}$ for DG reactive power injections, and $\mathbf{V}(k)=$ $\left[V_{1}(k), \ldots, V_{N_{\text {bus }}}(k)\right]^{T}$, where $N_{\text {tap }}, \quad N_{\mathrm{CB}}, \quad N_{\mathrm{DG}} \quad$ and $N_{\text {bus }}$ are the number of tap changers, CBs, DGs and buses, respectively. Accordingly, the incremental vectors $\Delta(\bullet)$ and minimum/maximum vectors $\overline{(\bullet)},(\bullet)$ can be defined to be consistent with the vectors above.

\section{A. Slow-Time-Scale Control}

In the STC, in addition to voltage regulation, the switching operations of OLTC, SVR and CBs with slow dynamics are optimized. The impact of active power variations of DGs can be taken into account via short-term forecasting. Furthermore, the voltage control scheme is developed based on the scenario that DGs track the maximum power point and the corresponding available reactive power capability of DGs is considered. It is to capture more energy while maintaining the voltage within the feasible range.

The MPC problem can be formulated as,

$$
\begin{aligned}
& \underset{\mathbf{n}_{\text {tap }}, \mathbf{n}_{\mathrm{CB}}, \mathbf{Q}_{\mathrm{DG}}, \mathbf{V}}{\operatorname{minimize}} \sum_{k=1}^{N_{p}^{\mathrm{STC}}}\left(\left\|\mathbf{n}_{\mathrm{tap}}(k)-\mathbf{n}_{\text {tap }}(k-1)\right\|_{\mathbf{C}_{\text {tap }}}^{2}\right. \\
&\left.+\left\|\mathbf{n}_{\mathrm{CB}}(k)-\mathbf{n}_{\mathrm{CB}}(k-1)\right\|_{\mathbf{C}_{\mathrm{CB}}}^{2}+\left\|\mathbf{V}(k)-\mathbf{V}_{\text {ref }}\right\|_{\mathbf{C}_{\mathrm{V}}}^{2}\right)
\end{aligned}
$$

subject to

$$
\begin{aligned}
& \underline{\mathbf{n}}_{\text {tap }} \leq \mathbf{n}_{\text {tap }}(k) \leq \overline{\mathbf{n}}_{\text {tap }}, \mathbf{n}_{\text {tap }} \in \mathbb{Z}^{N_{\text {tap }}, \forall k} \\
& \Delta \underline{\mathbf{n}}_{\text {tap }} \leq \Delta \mathbf{n}_{\text {tap }}(k) \leq \Delta \overline{\mathbf{n}}_{\text {tap }}, \forall k \\
& 0 \leq \mathbf{n}_{\mathrm{CB}}(k) \leq \overline{\mathbf{n}}_{\mathrm{CB}}, \mathbf{n}_{\mathrm{CB}} \in \mathbb{Z}^{N_{\mathrm{CB}}}, \forall k \\
& \Delta \underline{\mathbf{n}}_{\mathrm{CB}} \leq \Delta \mathbf{n}_{\mathrm{CB}}(k) \leq \Delta \overline{\mathbf{n}}_{\mathrm{CB}}, \forall k \\
& \underline{\mathbf{Q}}_{\mathrm{DG}}(k) \leq \mathbf{Q}_{\mathrm{DG}}(k) \leq \overline{\mathbf{Q}}_{\mathrm{DG}}(k), \forall k \\
& \mathbf{V}(k)=\mathbf{V}(0)+\frac{\partial \mathbf{V}}{\partial \mathbf{n}_{\text {tap }}^{T}} \Delta \mathbf{n}_{\mathrm{tap}}(k)+\frac{\partial \mathbf{V}}{\partial \mathbf{n}_{\mathrm{CB}}^{T}} \Delta \mathbf{n}_{\mathrm{CB}}(k) \\
&+\frac{\partial \mathbf{V}}{\partial \mathbf{Q}_{\mathrm{DG}}^{T}} \Delta \mathbf{Q}_{\mathrm{DG}}(k)+\frac{\partial \mathbf{V}}{\partial \mathbf{P}_{\mathrm{DG}}^{T}} \Delta \overline{\mathbf{P}}_{\mathrm{DG}}(k), \forall k
\end{aligned}
$$

where $\mathbf{V}_{\text {ref }}$ is the voltage reference, which is typically 1.0 p.u. and $\mathbf{V}(0)$ represents the measured voltage magnitude at the current operating point. Since a unified voltage profile could also result in network loss minimization [21], we select the unified voltage reference for all buses. $\mathbf{C}_{\mathrm{tap}}, \mathbf{C}_{\mathrm{CB}}$, and $\mathbf{C}_{\mathrm{V}}$ are the weighting matrices corresponding to the three terms. $\overline{\mathbf{P}}_{\mathrm{DG}}$ is the forecast available power of DGs and $\Delta \overline{\mathbf{P}}_{\mathrm{DG}}=\overline{\mathbf{P}}_{\mathrm{DG}}-\mathbf{P}_{\mathrm{DG}}(0)$. The reactive power limit of a DG unit is estimated by $\bar{Q}_{\mathrm{DG}}=\sqrt{S_{\mathrm{DG}}^{2}-P_{\mathrm{DG}}^{2}}$ and $\underline{Q}_{\mathrm{DG}}=-\sqrt{S_{\mathrm{DG}}^{2}-P_{\mathrm{DG}}^{2}}$, where $S_{\mathrm{DG}}$ is the rating capacity. $\partial \mathbf{V} / \partial \mathbf{n}_{\text {tap }}, \partial \mathbf{V} / \partial \mathbf{n}_{\mathrm{CB}}, \partial \mathbf{V} / \partial \mathbf{P}_{\mathrm{DG}}$, and $\partial \mathbf{V} / \partial \mathbf{Q}_{\mathrm{DG}}$ are the voltage sensitivity coefficient matrices w.r.t tap changes, actions of CBs, active/reactive power injections of DGs. These matrices are updated in each FTC period using the analytical method presented in Section IV. In the cost function, the first and second terms are used to reduce the actions of tap changers and CBs. The third term aims to mitigate the voltage deviations.

By substituting the constraints (1g) into the cost function (1a) and reformulating the constraints (1b)-(1f), the problem of the STC can be transformed into the standard MIQP problem w.r.t the following multi-step decision variables $\mathbf{x}_{\mathrm{STC}}$ :

$$
\begin{aligned}
& \mathbf{u}_{\mathrm{STC}}(k):=\left[\Delta n_{\mathrm{tap}, 1}(k), \ldots, \Delta n_{\mathrm{tap}, N_{\mathrm{tap}}}(k), \Delta n_{\mathrm{CB}, 1}(k), \ldots,\right. \\
&\left.\Delta n_{\mathrm{CB}, N_{\mathrm{CB}}}(k), \Delta Q_{\mathrm{DG}, 1}(k), \ldots, \Delta Q_{\mathrm{DG}, N_{\mathrm{DG}}}(k)\right]^{T}, \forall k=1, \ldots, N_{p}^{\mathrm{STC}} \\
& \mathbf{x}_{\mathrm{STC}}:=\left[\mathbf{u}_{\mathrm{STC}}^{T}(1), \ldots, \mathbf{u}_{\mathrm{STC}}^{T}\left(N_{p}^{\mathrm{STC}}\right)\right]^{T}
\end{aligned}
$$

\section{B. Fast-Time-Scale Control}

In the FTC, the active and reactive power outputs of DGs are optimally controlled to deal with the voltage issues in the fast time scale (seconds to several minutes). The optimal control problem can be formulated as follows,

$$
\begin{aligned}
& \underset{\mathbf{P}_{\mathrm{DG}}, \mathbf{Q}_{\mathrm{DG}}, \mathbf{V}}{\operatorname{minimize}} \sum_{k=1}^{N_{p}^{\mathrm{FTC}}}\left(\left\|\mathbf{V}(k)-\mathbf{V}_{\mathrm{ref}}\right\|_{\mathbf{C}_{\mathrm{V}}}^{2}+\| \overline{\mathbf{P}}_{\mathrm{DG}}(k)-\right. \\
& \left.\mathbf{P}_{\mathrm{DG}}(k)\left\|_{\mathbf{C}_{\mathrm{cur}}}^{2}+\right\| \mathbf{Q}_{\mathrm{DG}}(k)-\mathbf{Q}_{\mathrm{DG}}(k-1) \|_{\mathbf{C}_{Q}}^{2}\right)
\end{aligned}
$$

subject to

$$
\begin{aligned}
0 & \leq \mathbf{P}_{\mathrm{DG}}(k) \leq \overline{\mathbf{P}}_{\mathrm{DG}}(k), \forall k \\
\underline{\mathbf{Q}}_{\mathrm{DG}}(k) & \leq \mathbf{Q}_{\mathrm{DG}}(k) \leq \overline{\mathbf{Q}}_{\mathrm{DG}}(k), \forall k \\
\mathbf{V}(k) & =\mathbf{V}(0)+\frac{\partial \mathbf{V}}{\partial \mathbf{P}_{\mathrm{DG}}^{T}} \Delta \mathbf{P}_{\mathrm{DG}}(k)+\frac{\partial \mathbf{V}}{\partial \mathbf{Q}_{\mathrm{DG}}^{T}} \Delta \mathbf{Q}_{\mathrm{DG}}(k), \forall k
\end{aligned}
$$

In (3a), the first term is used to correct the voltage deviations, the second term is used to penalize the active power curtailment of DGs and the third term is used to smoothen the reactive power variations of DGs. $\mathbf{C}_{\mathrm{V}}, \mathbf{C}_{\mathrm{cur}}$ and $\mathbf{C}_{Q}$ are the corresponding weighting matrices.

Similarly, the problem of the FTC can be transformed into the standard QP problem w.r.t the following multi-step decision variables $\mathrm{x}_{\mathrm{FTC}}$ :

$$
\begin{aligned}
\mathbf{u}_{\mathrm{FTC}}(k):=[ & \Delta P_{\mathrm{DG}, 1}(k), \ldots, \Delta P_{\mathrm{DG}, N_{\mathrm{DG}}}(k), \Delta Q_{\mathrm{DG}, 1}(k), \\
& \left.\ldots, \Delta Q_{\mathrm{DG}, N_{\mathrm{DG}}}(k)\right]^{T}, \forall k=1, \ldots, N_{p}^{\mathrm{FTC}} \\
\mathbf{x}_{\mathrm{FTC}}:= & {\left[\mathbf{u}_{\mathrm{FTC}}^{T}(1), \ldots, \mathbf{u}_{\mathrm{FTC}}^{T}\left(N_{p}^{\mathrm{FTC}}\right)\right]^{T} . }
\end{aligned}
$$

The FTC controller should consider the impact of updated control commands of tap changers and CBs on voltages when formulating the optimization problem (3).

\section{Sensitivity Computation}

The proposed voltage control scheme is based on the sensitivity (see (1d) and (3d)). Therefore, the voltage sensitivities should be updated in real time to accurately regulate the voltages. Firstly, the analytical sensitivity calculation method developed in [18] is adopted in this paper to compute the sensitivities w.r.t power injections $\partial V / \partial P$ and $\partial V / \partial Q$ and slack bus voltage $\partial V / \partial V_{S}$. Then, the voltage sensitivity w.r.t the control variables $n_{\mathrm{tap}}, n_{\mathrm{CB}}, P_{\mathrm{DG}}$ and $Q_{\mathrm{DG}}$ i.e., $\partial V / \partial P_{\mathrm{DG}}$, $\partial V / \partial P_{\mathrm{DG}}, \partial V / \partial n_{\text {tap }}^{\mathrm{OLTC}}, \partial V / \partial n_{\text {tap }}^{\mathrm{SVR}}$ and $\partial V / \partial n_{\mathrm{CB}}$ are calculated by extending the method in [18]. The sensitivity w.r.t the discrete control variables is approximated by the derivatives of the continuous relaxation. 


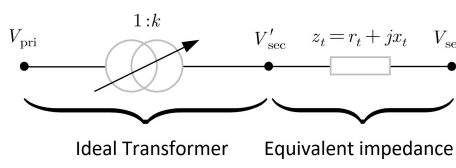

(a)

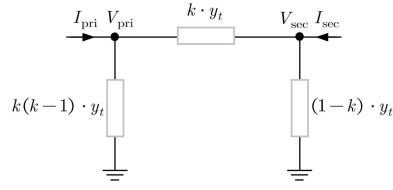

(b)
Fig. 2. Modeling of OLTC (a) Equivalent model of OLTC (b) $\pi$-equivalent model of a transformer $\left(y_{t}=1 / z_{t}=g_{t}+j b_{t}\right)$.

It is assumed that all the necessary information for the sensitivity calculation method is available via the communication infrastructures. An alternative approximate sensitivity computation method [22] can be used if only a limited number of bus voltages in the networks are monitored.

\section{A. Voltage Sensitivity With Respect to the Settings of CBs}

The reactive power injections of CBs can be expressed as,

$$
Q_{\mathrm{CB}}=n_{\mathrm{CB}} \cdot q_{\mathrm{CB}}
$$

where $q_{\mathrm{CB}}$ is the reactive power capacity of each unit of the CBs. Accordingly, the sensitivity coefficient of Bus $i$ with respect to $j$ th $\mathrm{CB}$ unit $\partial V_{i} / \partial n_{C B, j}$ can be calculated as,

$$
\frac{\partial V_{i}}{\partial n_{\mathrm{CB}, j}}=\frac{\partial V_{i}}{\partial Q_{\mathrm{CB}, j}} \cdot q_{\mathrm{CB}, j} \text {. }
$$

\section{B. Voltage Sensitivity With Respect to Tap Changes of OLTC}

The equivalent model of an OLTC transformer is shown in Fig. 2(a). Considering a transformer with OLTC on the secondary winding (MV side) as illustrated in Fig. 2(a), the ratio relationship can be expressed as,

$$
\frac{V_{\mathrm{sec}}^{\prime}}{V_{\mathrm{pri}}}=\left(1+n_{\mathrm{tap}}^{\mathrm{OLTC}} \cdot \Delta V_{\mathrm{tap}}^{\mathrm{OLTC}}\right) \cdot \frac{V_{N 1}}{V_{N 2}}
$$

where $V_{\mathrm{sec}}$ and $V_{\mathrm{pri}}$ are the primary and secondary-side voltages, respectively; $V_{\mathrm{sec}}^{\prime}$ is the virtual bus voltage; $V_{N 1}$ and $V_{N 2}$ are the nominal voltages; $n_{\text {tap }}^{\text {OLTC }}$ is the tap position and $\Delta V_{\text {tap }}^{\text {OLTC }}$ is the voltage step per tap; $z_{t}=r_{t}+\mathrm{j} x_{t}$ is the equivalent impedance of the transformer and $y_{t}=1 / z_{t}$. Suppose the slack bus voltage $V_{\text {pri }}$ keeps constant and the tap position moves from $n_{\text {tap }}^{\text {OLTC }}$ to $n_{\text {tap }}^{\text {OLTC }}$, the voltage sensitivity coefficients with respect to tap changes of the OLTC can be calculated by,

$$
\frac{\partial V_{i}}{\partial n_{\text {tap }}}=V_{\text {pri }} \cdot \Delta V_{\text {tap }}^{\text {OLTC }} \cdot \frac{V_{N 1}}{V_{N 2}} \cdot \frac{\partial V_{i}}{\partial V_{S}} .
$$

\section{Voltage Sensitivity With Respect to Tap Changes of SVR}

Since the SVR is often placed in the middle of a feeder, the primary side of the SVR cannot be considered as a slack bus anymore, implying that the calculation method for the OLTC might not be applicable for the SVR. Thus, an approximate calculation method is derived to deal with the issue. The SVR is basically a transformer, of which the equivalent $\pi$-model (see Fig. 2(b)) can be obtained from Fig. 2(a). The variations of $k y_{t}$ can be ignored since the ratio $k$ is always around the 1.0 p.u.. The tap changer can change the voltage profile mainly relying on the variations of the two parallel branches' admittance $y_{\text {pri }}=k(k-1) y_{t}$ and $y_{\mathrm{sec}}=(1-k) y_{t}$. Thus, the equivalent variations of power injections from these two branches can be expressed by,

$$
\begin{aligned}
& \Delta \widetilde{S}_{\mathrm{pri}}=V_{\mathrm{pri}}^{2} \cdot y_{\mathrm{pri}}^{*}-\left(V_{\mathrm{pri}}^{\prime}\right)^{2} \cdot y_{\mathrm{pri}}^{\prime *} \approx-\Delta k \cdot V_{\mathrm{pri}}^{2} \cdot y_{t}^{*}, \\
& \Delta \widetilde{S}_{\mathrm{sec}}=V_{\mathrm{sec}}^{2} \cdot y_{\mathrm{sec}}^{*}-\left(V_{\mathrm{sec}}^{\prime}\right)^{2} \cdot y_{\mathrm{sec}}^{\prime *} \approx \Delta k \cdot V_{\mathrm{sec}}^{2} \cdot y_{t}^{*}
\end{aligned}
$$

where $V_{\text {pri }}^{\prime}$ and $V_{\mathrm{sec}}^{\prime}$ denote the voltage after the tap actions. In the above derivations, the variations of voltage magnitude are ignored since they are generally around the reference.

Then, the variation of voltage at bus $i$ can be estimated by,

$$
\begin{aligned}
\Delta V_{i} & \approx \frac{\partial V_{i}}{\partial P_{\mathrm{pri}}} \cdot \operatorname{Re}\left(\Delta \widetilde{S}_{\mathrm{pri}}\right)+\frac{\partial V_{i}}{\partial Q_{\mathrm{pri}}} \cdot \operatorname{Im}\left(\Delta \widetilde{S}_{\mathrm{pri}}\right) \\
& +\frac{\partial V_{i}}{\partial P_{\mathrm{sec}}} \cdot \operatorname{Re}\left(\Delta \widetilde{S}_{\mathrm{sec}}\right)+\frac{\partial V_{i}}{\partial Q_{\mathrm{sec}}} \cdot \operatorname{Im}\left(\Delta \widetilde{S}_{\mathrm{sec}}\right) .
\end{aligned}
$$

Accordingly, $\partial V_{i} / \partial n_{\text {tap }}^{\mathrm{SVR}}$ can be calculated by,

$$
\begin{aligned}
\frac{\partial V_{i}}{\partial n_{\mathrm{tap}}^{\mathrm{SVR}}} & =\Delta V_{\mathrm{tap}}^{\mathrm{SVR}} \cdot\left(\frac{\partial V_{i}}{\partial Q_{\mathrm{pri}}} \cdot b_{t}-\frac{\partial V_{i}}{\partial P_{\mathrm{pri}}} \cdot g_{t}\right) \cdot V_{\mathrm{pri}}^{2} \\
& +\Delta V_{\mathrm{tap}}^{\mathrm{SVR}} \cdot\left(\frac{\partial V_{i}}{\partial P_{\mathrm{sec}}} \cdot g_{t}-\frac{\partial V_{i}}{\partial Q_{\mathrm{sec}}} \cdot b_{t}\right) \cdot V_{\mathrm{sec}}^{2},
\end{aligned}
$$

where the sensitivity coefficients $\partial V_{i} / \partial P_{\mathrm{pri}}, \partial V_{i} / \partial Q_{\mathrm{pri}}$, $\partial V_{i} / \partial P_{\mathrm{sec}}$, and $\partial V_{i} / \partial Q_{\mathrm{sec}}$ can be obtained based on the method in [18], respectively.

\section{Solution Method}

In recent years, the operator splitting method known as ADMM has received particular attention because of its good practical convergence behavior. It blends the decomposability of dual ascent with the superior convergence properties of the method-of-multipliers, which can reliably provide a modest accuracy solution in a handful number of iterations [23]. Furthermore, ADMM steps are computationally cheap and simple to implement, and thus it is ideal for embedded optimization. Clearly, the optimal control problems of the FTC, with continuous decision variables, can be efficiently solved by the ADMM algorithm. In the STC, the inclusion of discrete decision variables brings barriers to the solution of the problems. To this end, a tailored exact solution method based on the B\&B algorithm embedded with the ADMM-based QP solver is specially designed to solve the MIQP problem in real time.

\section{A. ADMM-Based Fast QP Solver}

1) Problem: Problem (1) of the FTC or problem (3) of the STC with continuous relaxations can be transformed into the standard QP problem as,

$$
\begin{array}{ll}
\underset{\mathbf{x}}{\operatorname{minimize}} & \frac{1}{2} \mathbf{x}^{T} \mathbf{H} \mathbf{x}+\mathbf{g}^{T} \mathbf{x} \\
\text { subject to } & \underline{\mathbf{x}} \leq \mathbf{x} \leq \overline{\mathbf{x}}
\end{array}
$$

where $\mathbf{x} \in \mathbb{R}^{n}$ denotes $\mathbf{x}_{\mathrm{STC}}$ or $\mathbf{x}_{\mathrm{FTC}}, \mathbf{H} \in \mathbb{R}^{n \times n}$ and $\mathbf{g} \in$ $\mathbb{R}^{n}$ denote the corresponding Hessian matrix and coefficient vector, respectively. For the STC, $n=N_{p}^{\mathrm{STC}} \times\left(N_{\mathrm{tap}}+N_{\mathrm{CB}}+\right.$ $\left.N_{\mathrm{DG}}\right)$ and for the FTC, $n=N_{p}^{\mathrm{FTC}} \times\left(N_{\mathrm{DG}}+N_{\mathrm{DG}}\right)$. 


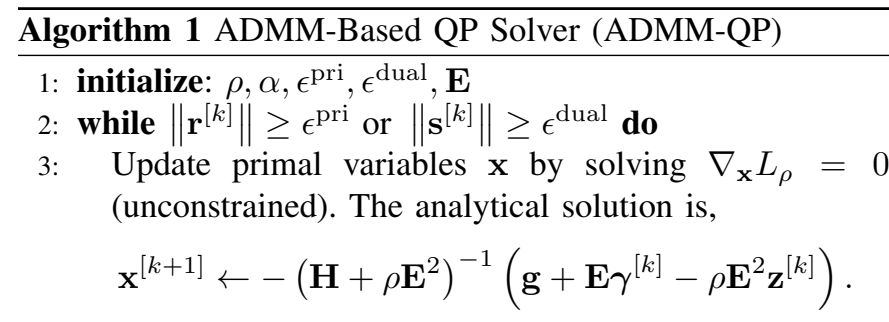

4: Update auxiliary variables $\mathbf{z}$ by,

$$
\begin{aligned}
& \mathbf{z}^{[k+1]} \leftarrow \Pi_{\mathcal{X}}\left(\tilde{\mathbf{x}}^{[k+1]}-\frac{1}{\rho} \mathbf{E}^{-1} \boldsymbol{\gamma}^{[k]}\right) \\
& =\min \left\{\max \left\{\tilde{\mathbf{x}}^{[k+1]}+\frac{1}{\rho} \mathbf{E}^{-1} \boldsymbol{\gamma}^{[k]}, \underline{\mathbf{x}}_{i}\right\}, \overline{\mathbf{x}}_{i}\right\}
\end{aligned}
$$

where $\tilde{\mathbf{x}}^{[k+1]}=\alpha \mathbf{x}^{[k+1]}+(1-\alpha) \mathbf{z}^{[k]}$.

5: Update dual variables $\gamma$ by,

$$
\gamma^{[k+1]}=\boldsymbol{\gamma}^{[k]}+\rho\left(\mathbf{E} \mathbf{x}^{[k+1]}-\mathbf{E} \mathbf{z}^{[k+1]}\right) .
$$

6: Update primal residual and dual residual,

$$
\begin{aligned}
& \mathbf{r}^{[k+1]} \leftarrow \mathbf{E} \mathbf{x}^{[k+1]}-\mathbf{E} \mathbf{z}^{[k+1]} \\
& \mathbf{s}^{[k+1]} \leftarrow \rho \mathbf{E}^{2}\left(\mathbf{z}^{[k+1]}-\mathbf{z}^{[k]}\right) .
\end{aligned}
$$

7: end while

2) Preconditioning: A known drawback of the firstorder methods is that they cannot effectively deal with illconditioned problems. In this paper, the decision variables, including the tap position of OLTC, settings of CBs and power outputs of DG show significantly different contributions to voltage regulation, which can be seen from the sensitivity coefficients, and the domains of these variables are in quite different scales. This will deteriorate the convergence of the ADMM algorithm. To this end, a diagonal matrix $\mathbf{E} \in \mathbb{R}^{n \times n}$ is introduced to improve the convergence by scaling the original decision variables, which can be found by minimizing the effective condition number of the following matrix $M$ [24],

$$
\mathbf{M}:=\mathbf{E H}^{\dagger} \mathbf{E} .
$$

where $\mathbf{H}^{\dagger}$ denotes the pseudo-inverse matrix of $\mathbf{H}$. Generally, the optimal solution of $\mathbf{E}$ can be found by solving a semidefinite programming problem, which is more complicated than solving the original QP problem and consequently computationally expensive for real-time control. Instead, to reduce the computation burden, the Ruiz Equilibration in $\infty$-norm is applied to compute the scaling matrix $\mathbf{E}$ [25].

3) ADMM Algorithm: Once the scaling matrix $\mathbf{E}$ is obtained, the preconditioned ADMM can be written as,

$$
\begin{aligned}
\underset{\mathbf{x}, \mathbf{z}}{\operatorname{minimize}} & \frac{1}{2} \mathbf{x}^{T} \mathbf{H} \mathbf{x}+\mathbf{g}^{T} \mathbf{x}+I_{\mathcal{X}}(\mathbf{z}) \\
\text { subject to } & \mathbf{E} \mathbf{x}-\mathbf{E} \mathbf{z}=\mathbf{0}, \\
& \mathbf{z} \in \mathcal{X}
\end{aligned}
$$

where $I_{\mathcal{X}}$ denotes the indicator function of $\mathcal{X}$, so that $I_{\mathcal{X}}(\mathbf{x})=$ 0 for $x \in \mathcal{X}$ and $I_{\mathcal{X}}(\mathbf{x})=\infty$ for $\mathbf{x} \notin \mathcal{X}$.

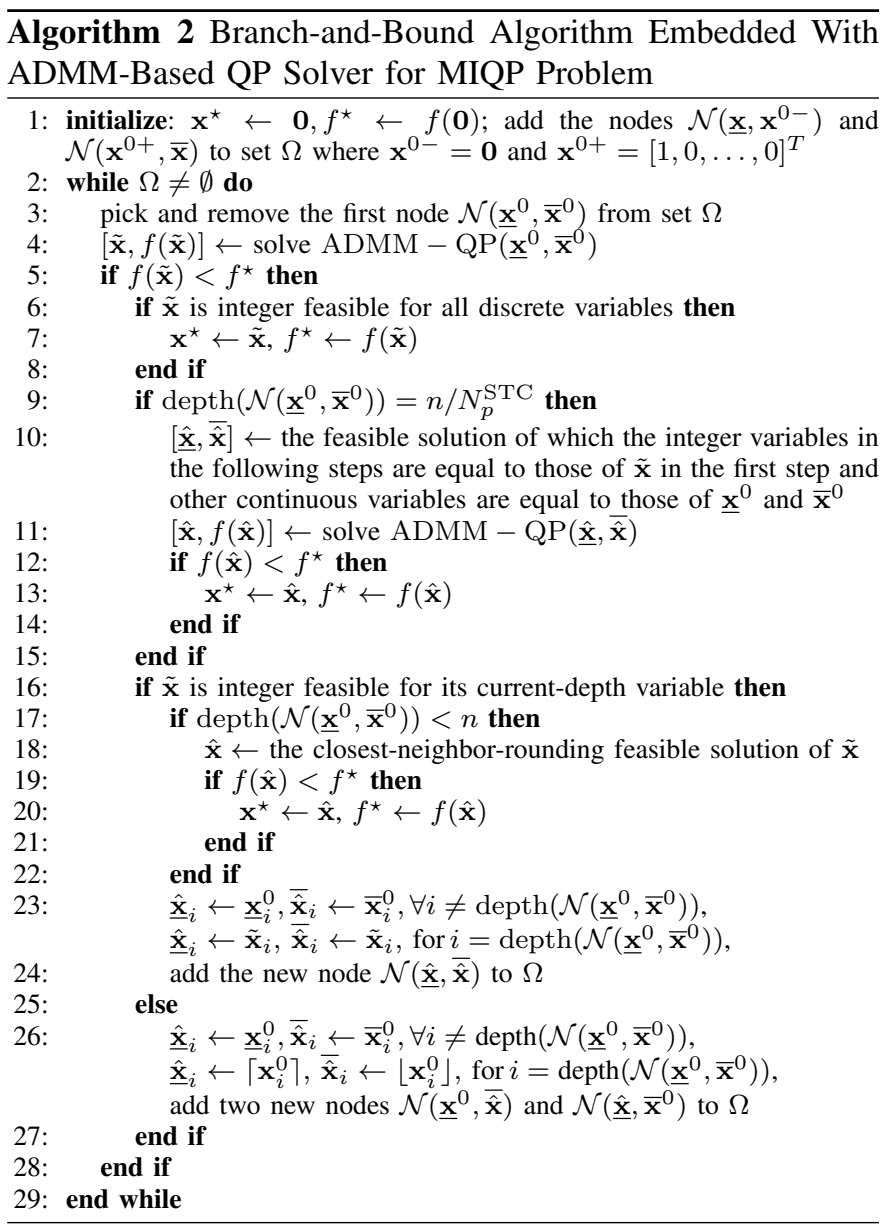

The augmented Lagrangian function for (14) is,

$$
\begin{aligned}
L_{\rho}(\mathbf{x}, \mathbf{z}, \gamma)= & \frac{1}{2} \mathbf{x}^{T} \mathbf{H} \mathbf{x}+\mathbf{g}^{T} \mathbf{x}+I_{\mathcal{X}}(\mathbf{z}) \\
& +\gamma^{T}(\mathbf{E x}-\mathbf{E} \mathbf{z})+\frac{\rho}{2}\|\mathbf{E} \mathbf{x}-\mathbf{E} \mathbf{z}\|_{2}^{2}
\end{aligned}
$$

where $\rho>0$ is the augmented Lagrangian parameter. In the ADMM algorithm, the augmented Lagrangian is minimized w.r.t the primal and auxiliary variables $\mathrm{x}$ and $\mathrm{z}$ sequentially. The dual variable $\gamma$ is updated using a step size equal to $\rho$. The detailed iteration method of the ADMM algorithm for the presented QP problem is presented in Algorithm 1.

The presented ADMM-based QP solver is directly applied to solve the optimal control problem of the FTC. Next, it will be embedded into the $\mathrm{B} \& \mathrm{~B}$ algorithm to find the optimal solution of the MIQP problem in the STC.

\section{B. Branch-and-Bound Algorithm Embedded With the ADMM- Based QP Solver for MIQP Problems in STC}

The B\&B algorithm [26]-[27] solves the MIQP problem by exploring the combinations of integer decision variables in the form of a tree. Each node of the tree denotes an optimization problem, with the sub-domain of the original problem and continuous relaxation on a part of discrete variables. To improve the efficiency, we aim to significantly reduce the number of visited nodes with the help of several heuristic strategies, specially designed as follows: 


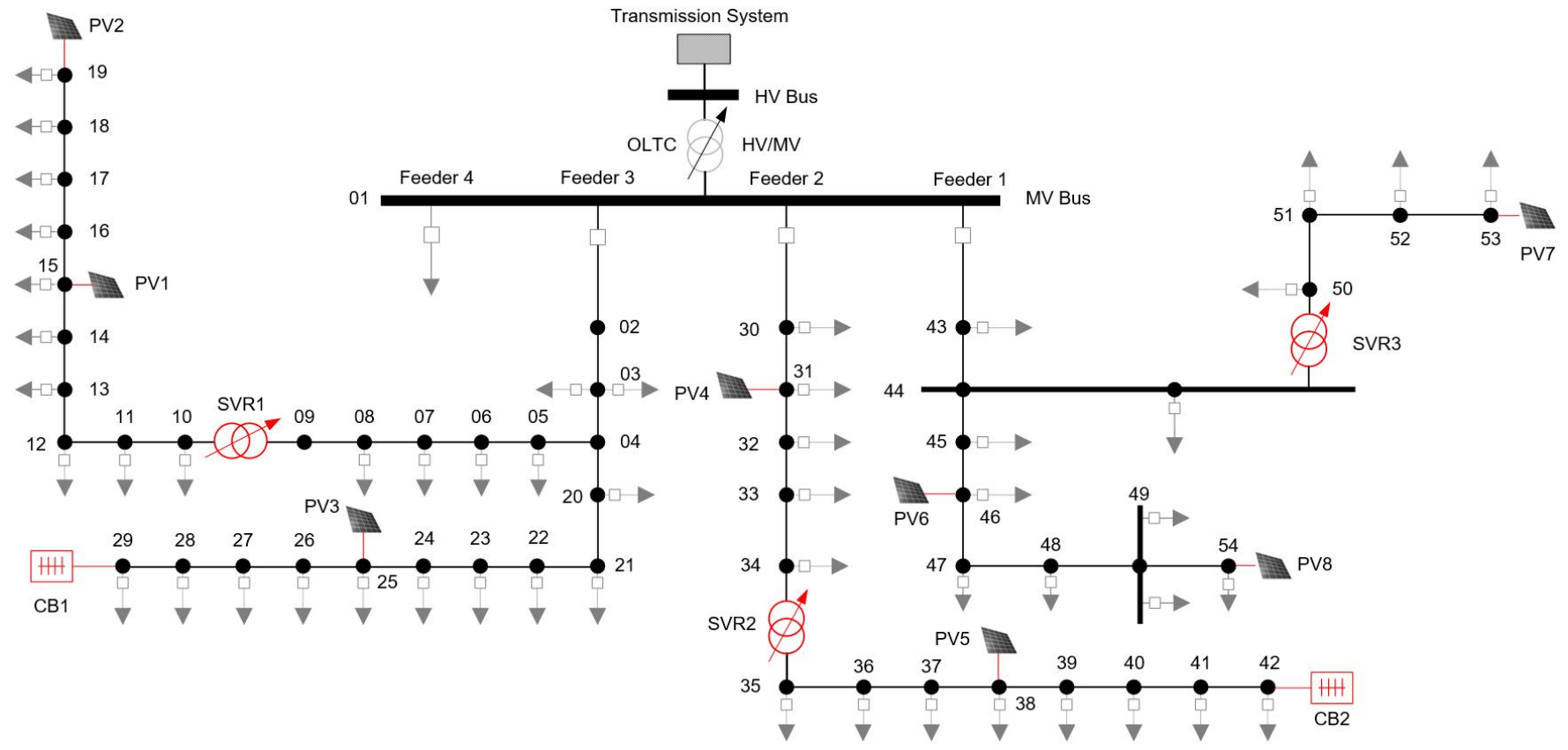

Fig. 3. Configuration and topology of the test system.

- Set $\mathbf{x}=\mathbf{0}$ as the initial guess of the optimal solution.

- When the depth of the current node (the number of the discrete decision variables that are already integer feasible) is equal to the number of the decision variables in one prediction step, a heuristic solution is developed by assigning the same value in the first step to the following steps and check/update the current upper bound.

- The nearest-neighbor-rounding strategy is used.

- The ADMM-based QP solver is warm-started with the solution of its parent node.

The breadth-first search is used in this algorithm. The pseudocode of the proposed method is presented in Algorithm 2. The corresponding notations of symbols are given as follows:

$\mathbf{x}^{\star}, f^{\star} \quad$ The current recorded optimal solution and the corresponding cost function value

$\mathcal{N}(\mathbf{a}, \mathbf{b}) \quad$ A node that represents (records) the constraint set $[\mathbf{a}, \mathbf{b}]$ in the search process

$\Omega \quad$ Pending node set

$\mathbf{x}, \overline{\mathbf{x}} \quad$ Lower/upper limits of $\mathbf{x}$

$\underline{x}^{0}, \overline{\mathbf{x}}^{0} \quad$ Lower/upper limits corresponding to the first node in the pending node set

$\mathbf{x}^{0-}, \mathbf{x}^{0+} \quad$ Auxiliary vectors that are used to initialize $\Omega$

$\tilde{\mathbf{x}}, f(\tilde{\mathbf{x}}) \quad$ The optimal solution and the corresponding cost function value by solving the QP problem with the constraint $\left\{\mathbf{x} \mid \underline{\mathbf{x}}^{0}<\mathrm{x}<\overline{\mathbf{x}}^{0}\right\}$

$\hat{\mathbf{x}}, \underline{\hat{\mathbf{x}}}, \overline{\hat{\mathbf{x}}} \quad$ Auxiliary vectors that are used for the mathematical description of the designed heuristic strategies

The operator $\mathrm{ADMM}-\mathrm{QP}(\mathbf{a}, \mathbf{b})$ means solving the $\mathrm{QP}$ problem with the constraint $\{\mathbf{x} \mid \mathbf{a} \leq \mathbf{x} \leq \mathbf{b}\}$ by the ADMMbased QP solver (Algorithm 1). The operator $\operatorname{depth}(\mathcal{N}(\mathbf{a}, \mathbf{b}))$ is used to get the depth of node $\mathcal{N}(\mathbf{a}, \mathbf{b})$, i.e. the number of elements of vectors $\mathbf{a}$ and $\mathbf{b}$ are integer feasible. Moreover,
TABLE I

PARAMETERS OF PV, OLTC, SVRS AND CBS

\begin{tabular}{lll}
\hline \hline Device & Location & Parameters \\
\hline PV1-PV8 & Bus15, 19,25,31,38,46,53,54 & $2 \mathrm{MW}$ \\
OLTC & HV Bus-MV Bus & $\pm 9 \times 1.67 \%$ \\
SVR1 & Bus09-Bus10 & $\pm 16 \times 0.625 \%$ \\
SVR2 & Bus34-Bus35 & $\pm 16 \times 0.625 \%$ \\
SVR3 & Bus44-Bus50 & $\pm 16 \times 0.625 \%$ \\
CB1 & Bus29 & $5 \times 100 \mathrm{kVar}$ \\
CB2 & Bus42 & $5 \times 100 \mathrm{kVar}$ \\
\hline
\end{tabular}

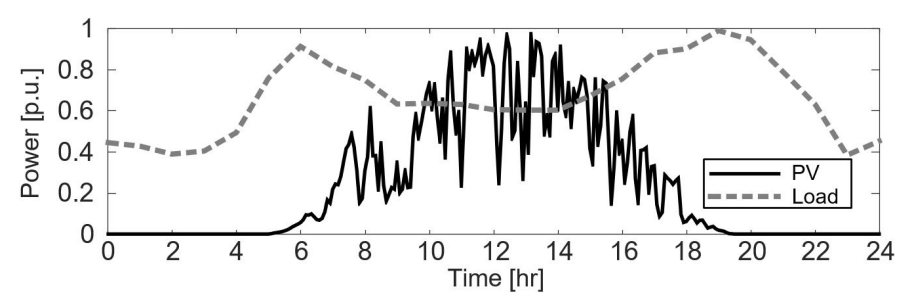

Fig. 4. Load and PV normalized profile.

for all vectors, the subscript $i$ denotes the $i$ th element of the vectors.

\section{NUMERICAL RESULTS}

\section{A. Basic Data and Parameters}

1) Network Model: A modified Italian $20 \mathrm{kV}$ distribution network with four feeders and a total of 54 buses is used in this paper, of which the topology and configuration are shown in Fig. 3. The network model parameters can be found in [9]. Eight PV units, three SVRs and two CBs are additionally placed into the original system. The installed capacity of PV, basic operation parameters of OLTC, SVRs, CBs and their corresponding placements are shown in Table I. 


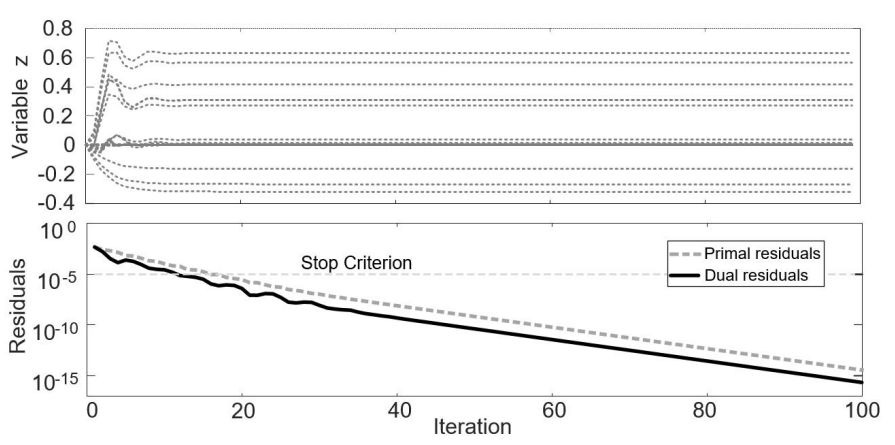

Fig. 5. Convergence rate with preconditioning.

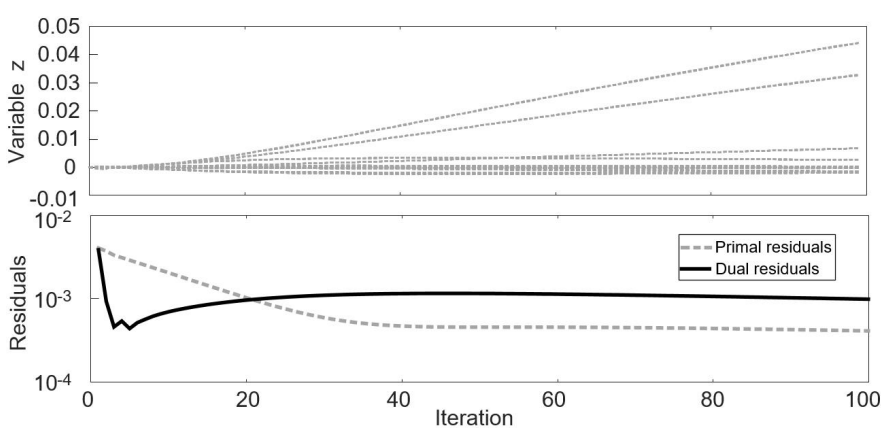

Fig. 6. Convergence rate without preconditioning.

2) Time Series of Loads and PV Outputs: The time-series of power loads is obtained by modifying an measured power load curve of the typical residential hourly load profile in the USA [28]. The time-series of available PV outputs is obtained from the National Renewable Energy Laboratory (NREL) Renewable Resource Data Center [29]. The normalized load and PV time series are shown in Fig. 4.

3) Control Parameters: The control periods of the STC and FTC are designed as $T_{c}^{\mathrm{STC}}=60 \mathrm{~min}$. and $T_{c}^{\mathrm{FTC}}=1 \mathrm{~min}$. and the number of prediction steps is designed as $N_{p}^{\mathrm{STC}}=3$ and $N_{p}^{\mathrm{FTC}}=10$, respectively. The convergence tolerances are set as $\epsilon_{\text {pri }}=\epsilon_{\text {dual }}=10^{-5}$, penalty parameter is set as $\rho=1$, and relaxation parameter is set as $\alpha=1.6$.

4) Implementation Platform: The proposed control scheme is implemented in the MATLAB R2016a and performed on a PC with an Intel(R) Core(TM) i7-6700HQ CPU @ 2.60GHz processor and 8 GB RAM, running Windows 10.

\section{B. Validation of the Solution Method}

1) Convergence Rate of ADMM: To illustrate the convergence performance of the ADMM algorithm, a snapshot of the STC during operation is selected to show the convergence process (see Figs. 5 and 6). It can be seen that the ADMM algorithm with preconditioning shows good convergence. It can converge to the optimal solution after about 20 iterations. However, without preconditioning, the primal and dual residuals decrease slightly after 40 iterations and it fails to converge within 100 iterations, indicating poor convergence. This validates the necessity and effectiveness of the preconditioning in this problem.

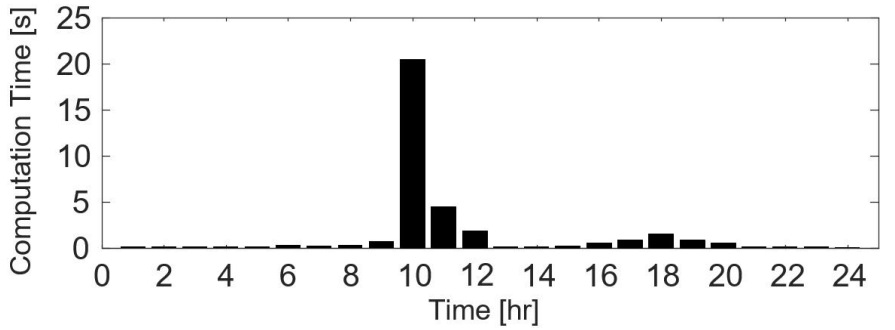

Fig. 7. Computation time of the STC at each control point.

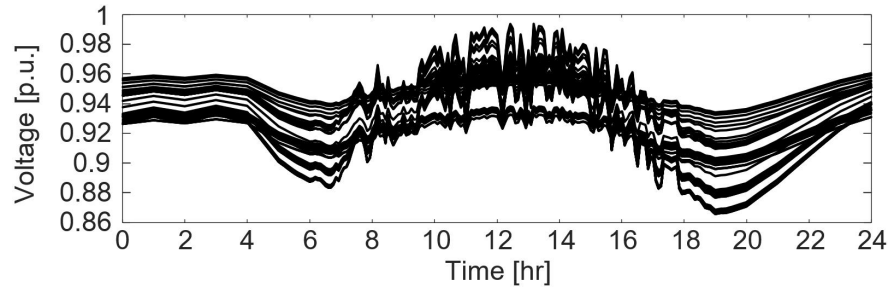

(a)

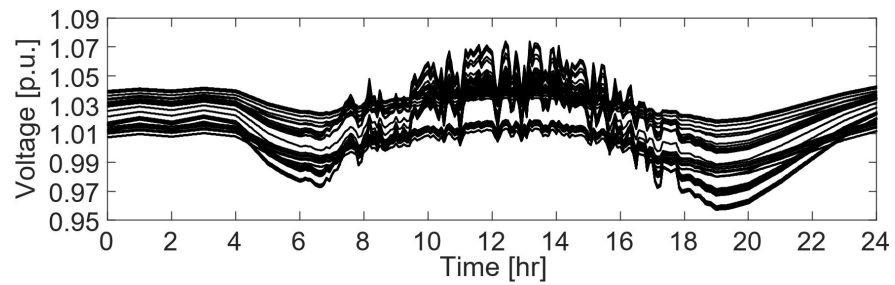

(b)

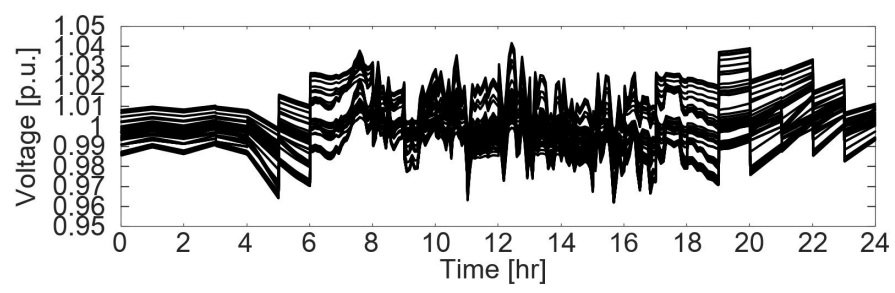

(c)

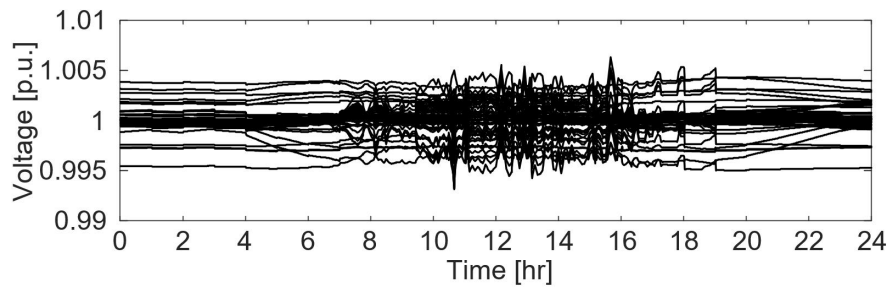

(d)

Fig. 8. Voltage profile only with (a) no real-time control $\left(n_{\text {tap }}^{\text {OLTC }}=0\right)$, (b) no real-time control $\left(n_{\mathrm{tap}}^{\mathrm{OLTC}}=5\right)$, (c) the STC and (d) the proposed doubles-time-scale control scheme,.

2) Efficiency: The computation time of the MIQP problems in the STC at each control point during the dynamic simulation is shown in Fig. 7. As can be seen from the figure, the computation time is mostly less than $2 \mathrm{~s}$. The longest computation time is about $20 \mathrm{~s}$. Compared with the control period of 60 min., the computation time is compatible with the real-time use. 


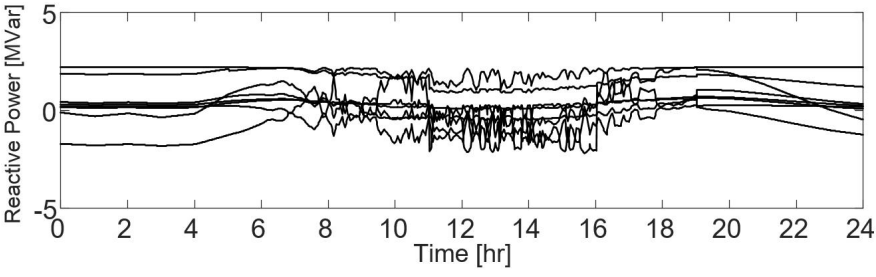

Fig. 9. Reactive power outputs of DGs.

TABLE II

SWITCHING OPERATION TIMES OF OLTC, SVRS AND CBS WITHIN A DAY

\begin{tabular}{lll}
\hline \hline Device & Slow-time-scale control & Double-time-scale control \\
\hline OLTC & 14 & 2 \\
SVR1 & 13 & 3 \\
SVR2 & 21 & 1 \\
SVR3 & 16 & 0 \\
CB1 & 8 & 2 \\
CB2 & 22 & 2 \\
\hline
\end{tabular}

\section{Effectiveness of the Proposed Voltage Control Scheme}

In Fig. 8, the voltage profile (obtained by the AC power flow calculation) across the network with the proposed controller is compared with profiles without control and only with the STC. Fig. 9 presents the reactive power outputs of DGs. Without any control (OLTC is set as $n_{\mathrm{tap}}^{\text {OLTC }}=0$ ), the voltage profile is much lower, with the most severe under-voltage occur during 18:00-20:00, approaching 0.86 p.u.(see Fig. 8(a)). If the lowest voltage is corrected to 0.95 p.u. (see Fig. $8(\mathrm{~b}), n_{\text {tap }}^{\text {OLTC }}=5$ ), the over-voltage will occur during 10:00-16:00, as the PV outputs are very high. It can be observed that the proposed control scheme can effectively regulate the voltage within the feasible range of 0.99-1.01 p.u.. By comparison, as shown in Fig. 8(a), the voltages without control significantly fluctuate along with the fluctuations of load and PV outputs. During 18:00-20:00, several bus voltages are even lower than 0.9 p.u., indicating the severe voltage deviations. As shown in Fig. 8(c), if only with the help of the STC, all bus voltages can also be effectively regulated within the range of $0.95-1.05$ p.u., relying on the tap changers and CBs. However, as shown in Table II, the tap changers and CBs are frequently adjusted, which will reduce the lifetime of the devices. Suppose the cost coefficients associated with the switching operation of tap changers and CB units are set as $1.40 \$ /$ time and $0.24 \$$ /time [10], the costs with the STC and the double-time-scale control are $96.8 \$$ day and $9.36 \$$ day, respectively. This validates the cost effectiveness of the double-time-scale control. The presence of the FTC can significantly not only correct the voltage deviations but also relieve the stress on tap changers and CBs.

\section{Comparison with the Continuous-Relaxation-Based Method}

As presented in the Section I, to simplify the problem and reduce the computational burdens of the controller, the discrete decision variables related to OLTC, SVRs, and CBs are often treated as continuous variables in the optimization model, i.e. the continuous-relaxation-based method. Here, the comparison

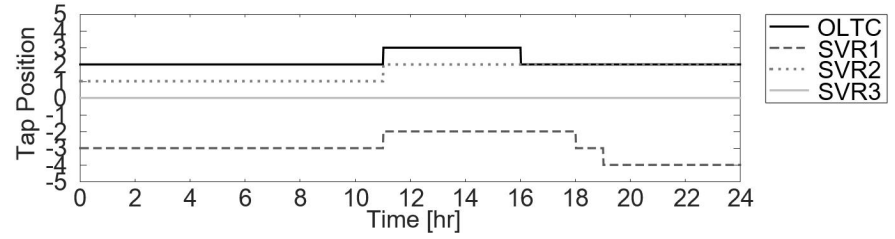

(a)

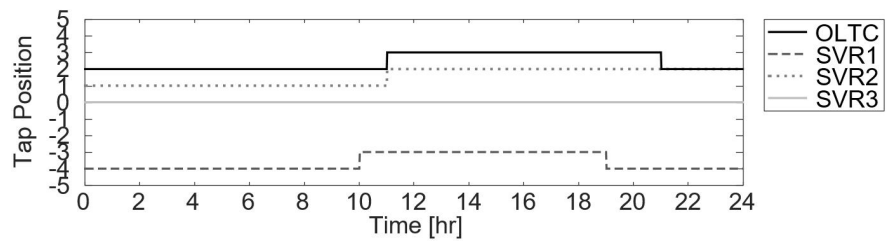

(b)

Fig. 10. Tap operations with (a) the proposed solution method and (b) continuous-relaxation-based method.

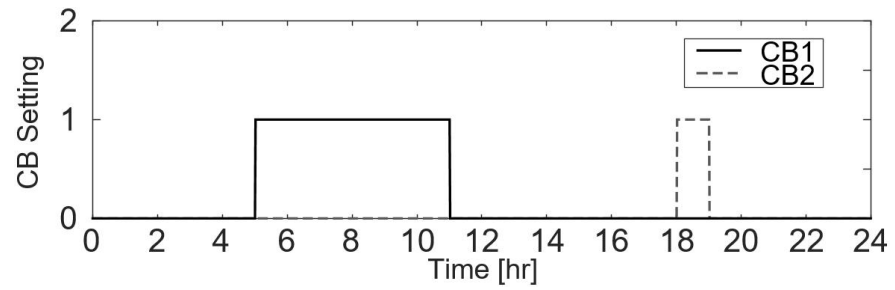

(a)

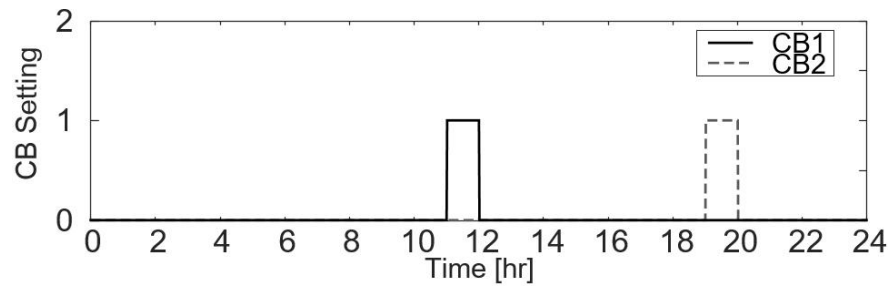

(b)

Fig. 11. CB setting with (a) the proposed solution method and (b) continuousrelaxation-based method.

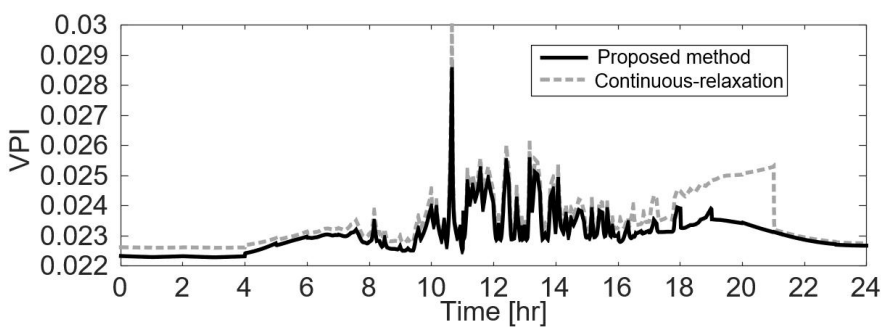

Fig. 12. Comparison of voltage control performance with the two different methods.

between the continuous-relaxation-based control method and the proposed method is provided.

The operations of OLTC, SVRs and CBs are presented in Figs. 10-11. The results of the two methods are different, implying that the continuous-based-relaxation method fails to obtain the optimal solution. In this case, though the accumulated switching operation times of OLTCs, SVRs and CBs are similar with the two methods, the time instants of the operations are different, which has an impact on voltage 


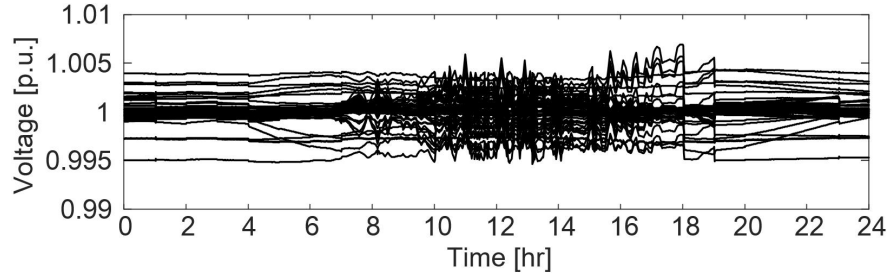

(a)

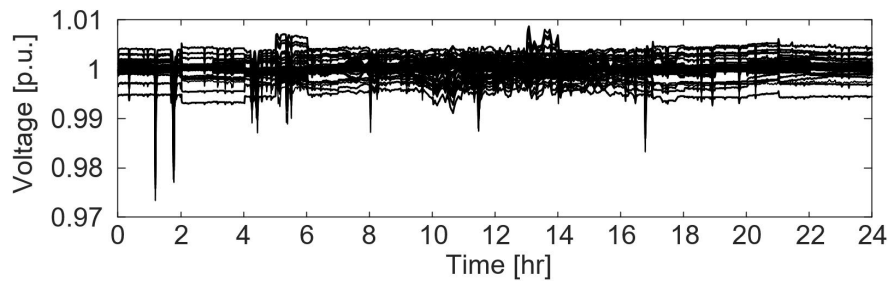

(b)

Fig. 13. Voltage control performance under the corrupted measurements with (a) $\sigma=5 \%$ (b) $\sigma=10 \%$.

performance. We define the voltage performance index by,

$$
V P I=\sum_{t=1}^{24 \times 60}\left\|\mathbf{V}(t)-\mathbf{V}_{\text {ref }}\right\| .
$$

As illustrated in Fig. 12, the $V P I$ with the proposed method is lower than the continuous-relaxation-based method, especially during 16:00-21:00, implying better control performance.

\section{E. Robustness}

In this subsection, the robustness of the proposed voltage control scheme against the corrupted measurements is tested. The measurement errors are assumed to follow the normal distribution,

$$
\hat{M}=(1+\varepsilon) M, \varepsilon \sim N\left(0, \sigma^{2}\right)
$$

where $M$ and $\hat{M}$ denote the actual value and measurement of voltages, powers, or line impedances, $\varepsilon$ denotes the relative error and $\sigma$ denotes the standard deviation, respectively. Fig. 13 shows the voltage profile with the proposed control method considering the corrupted measurements with $\sigma=5 \%$ and $10 \%$, respectively. As can be seen, the proposed controller can efficiently regulate the voltage within the feasible range 0.99 1.01 p.u. when $\sigma=5 \%$ and 0.97-1.01 p.u. when $\sigma=10 \%$, implying the good robustness.

\section{CONCLUSION}

In this paper, a double-time-scale voltage control scheme based on the MPC is proposed to coordinate voltage regulation devices with different time scales. An ADMM-based fast QP solution method is developed, which is directly used for solving the problems in the FTC. Then, a tailored exact solution method for the MIQP problem in the STC is developed based on the B\&B method embedded with the ADMM-based QP solver. The simulation results validate the effectiveness of the proposed voltage control scheme, which shows better control performance than only with the STC. Compared with the continuous-relaxation-based method, the developed solution method can achieve optimality. Furthermore, the computational efficiency of the proposed solution method is validated to be feasible for real-time use. Since LV distribution networks are often unbalanced with threephase four wires, the proposed voltage control scheme will be extended to handle the unbalanced voltage deviations in future work.

\section{REFERENCES}

[1] P. Paliwal, N. P. Patidar, and R. K. Nema, "Planning of grid integrated distributed generators: A review of technology, objectives and techniques," Renew. Sustain. Energy Rev., vol. 40, pp. 557-570, Dec. 2014.

[2] D. Zhang, J. Lin, and D. Hui, "Coordinated voltage control for voltage regulation of distribution network voltage regulation by distributed energy storage systems," Protection and Control of Modern Power Systems, vol. 3, no.3, pp. 35-42, May 2018.

[3] M. H. J. Bollen and A. Sannino, "Voltage control with inverter-based distributed generation," IEEE Trans. Power Delivery, vol. 20, no. 1, pp. 519-520, Jan. 2005.

[4] V. Calderaro, G. Conio, V. Galdi, G. Massa, and A. Piccolo, "Optimal decentralized voltage control for distribution systems with inverter-based distributed generators," IEEE Trans. Power Syst., vol.29, no. 1, pp. 230241, Jan. 2014

[5] N. Mahmud and A. Zahedi, "Review of control strategies for voltage regulation of the smart distribution network with high penetration of renewable distributed generation," Renew. Sustain. Energy Rev., vol. 64, pp. 582-595, 2016.

[6] A. Kulmala, S. Repo, and P. Jarventausta, "Coordinated voltage control in distribution networks including several distributed energy resources," IEEE Trans. Smart Grid, vol. 5, no. 4, pp. 2010-2020, Jul. 2014.

[7] Y. P. Agalgaonkar, B. C. Pal, and R. A. Jabr, "Distribution voltage control considering the impact of PV generation on tap changers and autonomous regulators," IEEE Trans. Power Syst., vol. 29, no. 1, pp. 182-192, Jan. 2014.

[8] B. A. Robbins, H. Zhu, and A. D. Dominguez-Garcia, ”Optimal tap setting of voltage regulation transformers in unbalanced distribution systems," IEEE Trans. Power Syst., vol. 31, no. 1, Apr. 2016.

[9] V. Calderaro, V. Galdi, F. Lamberti, and A. Piccolo, "A smart strategy for voltage control ancillary service in distribution networks," IEEE Trans. Power Syst., vol. 30, no. 1, pp. 494-502, Jan. 2015.

[10] P. Li, H. Ji, C. S. Wang, J. Zhao, G. Song, F. Ding, and J. Wu, "Coordinated control method of voltage and reactive power for active distribution networks based on soft open point," IEEE Trans. Sustain. Energy, vol. 8, no. 4, pp. 1430-1442, Oct. 2017.

[11] Y. J. Kim, J. L. Kirtley, and L. K. Norford, "Reactive power ancillary service of synchronous DGs in coordination with voltage control devices," IEEE Trans. Smart Grid, vol. 8, no. 2, pp. 515-527, Mar. 2017.

[12] Y. Zheng, D. J. Hill, K. Meng and S. Y. Hui,"Critical bus voltage support in distribution systems with electric springs and responsibility sharing", IEEE Trans. Power Syst., vol. 32, no. 5, pp. 3584-3593, Sep. 2017.

[13] M. Chertkov, D. Deka, and Y. Dvorkin, "Optimal ensemble control of loads in distribution grids with network constraints," pp. 1-7, Oct. 2017. [online].Available:http://arxiv.org/abs/1710.09924.

[14] K. Christakou, D.-C. Tomozei, J.-Y. Le Boudec, and M. Paolone, "GECN: Primary voltage control for active distribution networks via realtime demand-response," IEEE Trans. Smart Grid, vol. 5, no. 2, pp. 622 631, Mar. 2014.

[15] K. Christakou, D.-C. Tomozei, M. Bahramipanah, J.-Y. Le Boudec, and M. Paolone, "Primary voltage control in active distribution networks via broadcast signals: The case of distributed storage," IEEE Trans. Smart Grid, vol. 5, no. 5, pp. 2314-2325, Sep. 2014.

[16] K. Christakou, M. Paolone, and A. Abur, "Voltage control in active distribution networks under uncertainty in the system model: a robust optimization approach," IEEE Trans. Smart Grid, in press.

[17] G. Valverde, and T. V. Cutsem, "Model Predictive Control of voltages in active distribution networks," IEEE Trans. Smart Grid, vol. 4, no. 4, pp. 2152-2161, Dec. 2014.

[18] K. Christakou, J. LeBoudec, M. Paolone, and D.-C. Tomozei, "Efficient computation of sensitivity coefficients of node voltages and line currents in unbalanced radial electrical distribution networks," IEEE Trans. Smart Grid, vol. 4, no. 2, pp. 741-750, Jun. 2013. 
[19] K. E. Antoniadou-plytaria, I. N. Kouveliotis-lysikatos, P. S. Georgilakis, and N. D. Hatziargyriou, "Distributed and Decentralized Voltage Control of Smart Distribution Networks: Models, Methods , and Future Research," IEEE Trans. Smart Grid, vol. 8, no. 6, pp. 2999-3008, 2017.

[20] M. Glavic, M. Hajian, W. Rosehart, "Receding-horizon multi-step optimization to correct nonviable or unstable transmission voltages," IEEE Trans. on Power Syst., vol. 26, no. 3, pp. 1641-1650, Aug. 2011.

[21] A. Maknouninejad and Z. Qu,"Realizing unified microgrid voltage profile and loss minimization: A cooperative distributed optimization and control approach, IEEE Trans. Smart Grid, vol. 5,no. 4, pp. 1621-1630, Apr. 2014.

[22] Y. Guo, Q. Wu, H. Gao, X. Chen, J. Østergaard, and H. Xin, "MPCBased Coordinated Voltage Regulation for Distribution Networks With Distributed Generation and Energy Storage System," IEEE Trans. Sustain. Energy, in press.

[23] S. Boyd, N. Parikh, E. Chu, B. Peleato, and J.Eckstein, "Distributed optimization and statistical learning via the alternating direction method of multipliers," Found. Trends Mach. Learn., vol. 3, no. 1, pp. 1-122, 2011.

[24] R. Takapoui, N. Moehle, S. Boyd, Bemporad, and A. Bemporad, "A simple effective heuristic for embedded mixed-integer quadratic programming," International Journal of Control, pp. 1-11, 2017.

[25] D. Ruiz, "A scaling algorithm to equilibrate both rows and columns norms in matrices," Rutherford Appleton Lab., Oxfordshire, U.K., Tech. Rep. RAL-TR-2001-034, Oct. 2001.

[26] S. Boyd and J. Mattingley,"Branch and bound methods," Lecture notes, Stanford University, 2007

[27] L. G. Mitten, "Branch-and-bound methods: general formulation and properties," Operations Research, Vol. 18, No. 1, pp. 24-34, Feb. 1970.

[28] Commercial and residential hourly load profiles for all TMY3 locations in the United States, [Online] Available:http://openei.org/datasets/.

[29] Renewable resource data center, [Online] Available: https: // www.nrel. gov/rredc/.

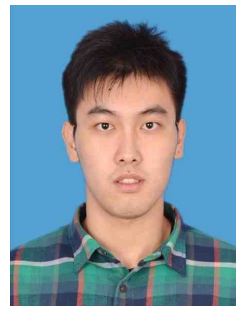

Yifei Guo received the B.E. in electrical engineering from Shandong University, Jinan, China, in 2014. Currently, he is pursuing the Ph.D. degree in electrical engineering at Shandong University. During 2017-2018, he was a visiting student in the Department of Electrical Engineering, Technical University of Denmark, Kgs. Lyngby, Denmark.

His research interests include, voltage control, wind power integration, power system protection, and distributed optimization and control algorithms with applications to power system,

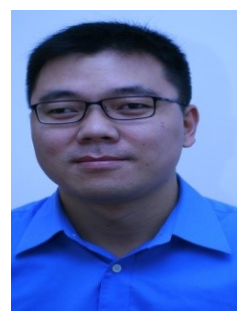

Qiuwei Wu (M'08-SM'15) obtained obtained the $\mathrm{PhD}$ degree in Power System Engineering from Nanyang Technological University, Singapore, in 2009. He was a senior R\&D engineer with VESTAS Technology R\&D Singapore Pte Ltd from Mar. 2008 to Oct. 2009. He is an Associate Professor at Department of Electrical Engineering, Technical University of Denmark (DTU) since Nov. 2009. He was a visiting scholar at Department of Industrial Engineering \& Operations Research (IEOR), University of California, Berkeley, from Feb. 2012 to May 2012 funded by Danish Agency for Science, Technology and Innovation (DASTI), Denmark. He was a visiting scholar at School of Engineering and Applied Sciences, Harvard University from Nov. 2017 to Oct. 2018.

His research area is power system operation and control with high renewables, including wind power modelling and control, active distribution networks, and integrated energy systems. He is an Editor of IEEE Transactions on Smart Grid and IEEE Power Engineering Letters. He is also an Associate Editor of International Journal of Electrical Power and Energy Systems, Journal of Modern Power Systems and Clean Energy, IET Renewable Power Generation, and IET Generation, Transmission \& Distribution.

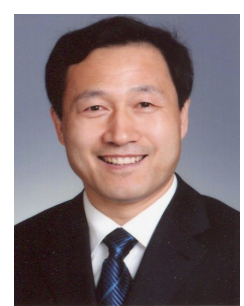

Houlei Gao (M'98) was born in Shandong, China, in 1963. He received the B.Sc. and M.Sc. degrees in electrical power engineering from Shandong University, Jinan, China, in 1983 and 1988, respectively, and the Ph.D. degree from Tianjin University, Tianjin, China, in 1997. From 2004 to 2005, he was with the School of Electrical and Electronic Engineering, Queen's University Belfast, Belfast, U.K. He is a Professor at the School of Electrical Engineering, Shandong University. His research interests include power system protection, feeder automation, distributed generation, and digital substation.

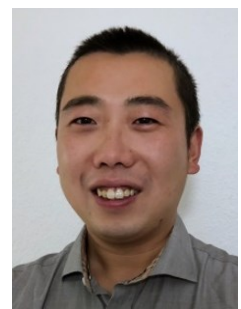

Sheng Huang received the M.S. and Ph.D. degree both in College of Electrical and Information Engineering, Hunan University, Changsha, China, in 2012 and 2016, respectively. He is currently a Postdoc with the Center for Electric Power and Energy, Department of Electrical Engineering, Technical University of Denmark. His research interests include renewable energy generation, modeling and integration study of wind power, control of energy storage system, and voltage control.

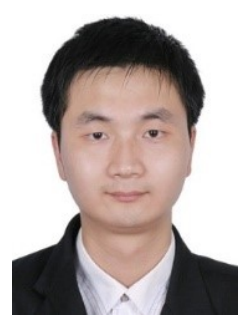

Bin Zhou (S'11-M'13-SM'17) was born in Hunan Province, China, in 1984. He received the B.Sc. degree in electrical engineering from Zhengzhou University, Zhengzhou, China, in 2006, the M.S. degree in electrical engineering from South China University of Technology, Guangzhou, China, in 2009, and the Ph.D. degree from The Hong Kong Polytechnic University, Hong Kong, in 2013. Afterwards, he worked as a Research Associate and subsequently a Postdoctoral Fellow in the Department of Electrical Engineering of The Hong Kong Polytechnic University. Now, he is an Associate Professor in the College of Electrical and Information Engineering, Hunan University, Changsha, China. His main fields of research include smart grid operation and planning, renewable energy generation, and energy efficiency.

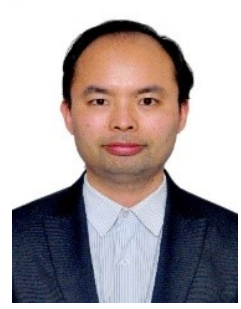

Canbing Li (M'06-SM'13) was born in Hunan Province, China, in 1979. He received the B.Sc. degree and the Ph.D. degree both in electrical engineering from Tsinghua University, Beijing, China, in 2001 and 2006, respectively. He is currently a Professor with the College of Electrical and Information Engineering, Hunan University, Changsha, China. His research interests include smart grid, energy efficiency and energy policy. 